\title{
La lingüística cognitiva: una aproximación al abordaje del lenguaje como fenómeno cognitivo integrado*
}

\author{
Javier Aníbal Moreno Mojica**
}

Recibido: 16 de junio de 2015 - Revisado: 23 de septiembre de 2015 -

Aprobado: 9 de noviembre de 2015

Resumen

La lingüística cognitiva es un movimiento lingüístico que concibe el lenguaje como una capacidad integrativa dentro de las tareas cognitivas propias del hombre, lo que devela que el lenguaje está basado en procesos de cognición complejos tales como la percepción, conceptualización, categorización, inferencia, entre otros, los que de una u otra forma permiten una aprehensión, funcionamiento, estructuración y adecuación de la realidad y el mundo. No obstante, debido a sus postulaciones en contraposición de paradigmas lingüísticos tradicionales, ha sido señalada más de teoría unificada que de una corriente como tal, llegando a desconocerse no solo sus orígenes, preguntas fundamentales, principios teóricos y tareas propias, sino sus aportes a la ciencia lingüística en lo que atañe a la concepción y comprensión de la facultad del lenguaje humano. A través de este artículo se presenta una aproximación a la lingüística cognitiva en cuanto a su desarrollo histórico, constructos teóricos fundamentales y tendencias particulares en lo propio a la relación lenguaje-cognición-realidad.

Palabras clave: lingüística cognitiva, lenguaje, cognición, comprensión, percepción, realidad.

* Artículo de reflexión.

** Maestría en Lingüística, UPTC. Especialización en Lingüística Aplicada a la Enseñanza del Inglés, UGC. Especialización en Docencia del Español como Lengua Propia, UPN. Teología, USTA. Licenciatura en Literatura y Lengua Castellana, USTA. Licenciatura en Filosofía, Pensamiento Político y Económico, USTA. Licenciatura en Idiomas Modernos EspañolInglés, UPTC. Se desempeña actualmente como decano de la Facultad de Teología de la Universidad Santo Tomás. Dirección postal: carrera 9 \# 63-28, Bogotá, Colombia, correo electrónico: javieranibalmoreno@gmail.com 


\title{
Cognitive linguistics: an approach to addressing language as a comprehensive cognitive phenomena*
}

\author{
Javier Aníbal Moreno Mojica**
}

Cognitive linguistics is a linguistic approach that studies and reflects on language as an integral capability within the human being's cognitive tasks. Due to that, language is based on complex cognition processes such as: perception, conceptualization, inference, etc., which allow getting a comprehension, a structuration and an adequacy of the reality. However, due to its particular issues as opposed to the traditional linguistic paradigms, this linguistic approach has been defined as a unified theory more than a specific theory itself. It has overlooked not only its origins, fundamental questions, theoretical principles and tasks, but its contributions to the linguistic science in relation to the conception and understanding of the human language faculty. This paper presents an introduction to the cognitive linguistics keeping in mind its historical development, fundamental theoretical frameworks, and particular trends on the relation given by language-cognition and reality.

Keywords: Cognitive linguistics, language, cognition, comprehension, perception, reality.

* Reflection article.

** Master in Linguistics, UPTC. Specialization in Linguistics Applied to Teaching English, UGC. Specialization in Teaching Spanish as Native Language, UPN. Theology, USTA. Degree in Spanish Literature and Language, USTA. Degree in Philosophy, Political and Economic Thinking, USTA. Degree in Modern Languages Spanish-English, UPTC. Currently serves as Dean of the Faculty of Theology at University of Santo Tomás. Postal address: carrera 9 \# 63-28, Bogotá, Colombia, e-mail: javieranibalmoreno@gmail.com 


\title{
La linguistique cognitive: une approche à l'abordage du langage comme un phénomène cognitif intégré*
}

\author{
Javier Aníbal Moreno Mojica**
}

\section{Résumé}

La linguistique cognitive est un mouvement linguistique qui conçoit le langage comme une capacité qui intègre, dans les tâches cognitives propres de l'homme, ce qui montre que le langage est basé sur des processus de cognition complexes tels que la perception, la conceptualisation, la catégorisation et la déduction, entre autres, lesquels d'une manière ou d'une autre permettent une appréhension, fonctionnement, structuration et adéquation de la réalité et du monde. Cependant, à cause de ses postulats en contradiction avec les paradigmes linguistiques traditionnels, cette théorie à davantage été qualifiée de théorie unifiée que considérée comme un courant comme tel, arrivant à en oublier non seulement ses origines, ses questions fondamentales, principes théoriques et tâches propres, mais aussi ses apports à la science linguistique en ce qui concerne la conception y compréhension de la faculté du langage humain. A travers cet article, il se présente une approche à la linguistique cognitive en rapport avec son développement historique, concepts théoriques fondamentaux et tendances particulières propre à la relation langage-cognition-réalité.

Mots clés: Linguistique cognitive, langage, cognition, compréhension, perception, réalité.

* Article de réflexion.

** Maîtrise en Linguistique, UPTC. Spécialisation en Linguistique Appliquée à I'Enseignement de I'Anglais, UGC. Spécialisation dans I'Enseignement de I'Espagnol comme langue maternelle, UPN. Théologie, USTA. Titulaire d'une Maîtrise en Littérature et Langue Espagnole, USTA. Titulaire d'une Maîtrise en Philosophie, Pensée Politique et Économique, USTA. Titulaire d'une Maîtrise en Langues Modernes Espagnol-Anglais, UPTC. Actuellement, il est recteur de la Faculté de Théologie de I'Université Santo Tomás. Adresse postale: carrera 9 \# 63-28, Bogota, Colombie, e-mail: javieranibalmoreno@gmail.com. 


\section{Introducción}

Atreverse a pensar en una corriente como la lingüística cognitiva hoy en día parece ser menos complejo de lo que pudo haberlo sido en el momento de mayor auge de los grandes paradigmas lingüísticos tradicionales, donde los postulados principales conducían a una concepción de lenguaje distante a la propuesta por esta corriente en la cual el lenguaje se fundamenta en una base experiencial dada a partir de la relación entre el mundo, la percepción y la cognición. Sin embargo, se evidencia aún cierto desconocimiento sobre la incidencia de los principios fundamentales y las tendencias de la lingüística cognitiva en el abordaje del fenómeno del lenguaje, y en la posibilidad de su tratamiento desde una óptica mucho más integradora, en donde su quehacer se registra en términos de interacción con el mundo y los procesos cognoscitivos que se llevan a cabo de forma relacional.

Asimismo, se ha concebido por parte de la lingüística hegemónica, que un modelo lingüístico, como el propuesto por la lingüística cognitiva, a pesar de ostentar un objeto de estudio y una metodología pertinentes, dejaba de lado los principios del innatismo, el modularismo y la lógica, - por solo citar algunos- y caía en un reduccionismo netamente funcional y experiencial, mostrando una debilidad mayúscula en el estudio del fenómeno del lenguaje humano, lo que conllevaba a que su labor se mostrara más como una limitación que como un aporte significativo en sí. Frente a este fundamento, y de acuerdo con Croft (2008), los cognitivistas respondieron desde tres hipótesis, que luego serían las postulaciones máximas de este movimiento, a saber: el lenguaje no constituye una facultad cognitiva autónoma, la gramática implica una conceptualización y el conocimiento sobre el lenguaje surge de su propio uso.

Teniendo en cuenta estas y otras posturas, con el presente texto se pretende brindar una aproximación, a modo de respuesta, a la cuestión: ¿por qué considerar la lingüística cognitiva un paradigma lingüístico funcional y experiencial en lo referente al abordaje del lenguaje como fenómeno cognitivo integrado?

Para lograr tal propósito se presentará como primer momento un breve esbozo histórico del surgimiento de la lingüística cognitiva. En la segunda parte, se señalarán someramente los fundamentos teóricos propuestos por esta corriente lingüística. En la tercera se abordarán, de manera sucinta, las principales tendencias de la lingüística cognitiva, y finalmente se esbozará un posible acercamiento a la lingüística cognitiva como paradigma funcional y experiencial en lo referente al abordaje del lenguaje como fenómeno cognitivo integrado.

\section{Orígenes de la lingüística cognitiva}

Para comprender la lingüística cognitiva en cuanto a sus inicios, Ibarretxe y Valenzuela (2012), señalan que es necesario reconocer el valor de los aportes 
realizados por las corrientes lingüísticas hegemónicas de la segunda mitad del siglo $\mathrm{XX}$, donde surge esta nueva perspectiva, en especial la del generativismo chomskiano, la cual criticó sobremanera la postura conductista hasta entonces imperante, y que fue capaz de "demostrar de manera irrefutable que era imposible explicar comportamientos humanos tan complejos como el lenguaje humano sin recurrir a constructos mentales" (p. 14). Con tal apreciación Chomsky no solo abrió las puertas al mentalismo en lo referente a la concepción y comprensión del lenguaje humano, sino que propuso los principios de innatismo y universalismo en lo que atañe a la capacidad lingüística humana.

Sin embargo, el marcado énfasis en los postulados del paradigma Chomskyano, a saber: el formalismo, la modularidad, la submolaridad, el carácter abstracto y las generalizaciones de alto nivel, relegó los aspectos funcionales del lenguaje en cuanto tratamiento del mismo, puesto que su atención se dirigió específicamente a los aspectos formales de este más que a su función.

Además de lo anterior, la teoría modularista del lenguaje sostenía como principio la existencia de un módulo cerebral autónomo dedicado específicamente a procesar información lingüística, lo que dejaba de lado la posibilidad de pensar en una integración relacional heterónoma en cuanto lenguaje y cerebro. Sumado a lo dicho, la tradición filosófica objetivista, por ende lógico-atomística, apartaba de su quehacer el proceso senso-perceptivo en las tareas de pensamiento, conceptualización, categorización de la realidad y del lenguaje mismo, puesto que el pensamiento como tal es susceptible de ser formalizado y lo mencionado lo impedía.

Adicional a ello, y siguiendo a Ibarretxe y Valenzuela (2012), "el generativismo ofrecía un modelo de lenguaje explícito formal y riguroso. Sin embargo dejaba de lado aspectos del lenguaje que algunos consideraban que eran imprescindibles para el estudio de la lengua, como los aspectos culturales, históricos, psicológicos, sociales o estilísticos" (p. 15). Pero sería el rechazo de incluir el significado, en los estudios y análisis del lenguaje por parte de los lingüistas del momento, lo que motivó a que varios estudiosos del lenguaje reaccionaran en contra de dicha postura, generándose así una propuesta paradigmática que partía de los presupuestos contrarios a los que la hegemonía lingüística planteaba.

Entonces, a mediados de la década de los setenta, un grupo de lingüistas dio inicio a una reflexión en torno al lenguaje, haciendo hincapié en el aspecto semántico del mismo, ya que tratar de explicar el lenguaje sin tener en cuenta el significado era complicado, y por ende llegar a suprimirlo sería extraer del análisis lingüístico un componente esencial en la explicación del mismo. De esta manera, figuras tales como George Lakoff, Charles Fillmore, Ronald Langacker, Leonard Talmy, entre otras, dieron apertura al paradigma lingüístico cognitivo, apoyados inicialmente por los desarrollos de la ciencia cognitiva, especialmente de la psicología y por los planteamientos contrarios al generativismo. 
Así, con las obras intituladas: Metaphors we live by de autoría de Lakoff y Johnson en 1980 y publicada en 1986 en castellano, Foundations of cognitive grammar de Langacker y Women, fire and dangerous things (mujeres, fuego y cosas peligrosas) de Lakoff, en 1987, el cual se basó en el trabajo de Dixón, se comenzó la nueva corriente lingüística, en tanto que esta última obra registra la categorización lingüística de las entidades del mundo desde un proceso experiencial propias del Dyirbal, una lengua indígena australiana, evidenciando lo que dichas categorías revelan sobre la organización de la mente, hasta el punto de mostrar "la existencia de una relación entre el lenguaje, el mundo y la cognición, que se puede sintetizar en algunos principios posiblemente comunes a los sistemas de categorización humana" (Cuenca, 2013, p. 27). Pero fue hasta 1989 con la celebración del primer congreso internacional realizado en Duisburgo (Alemania) que se inaugura como tal el nuevo paradigma dentro de la ciencia lingüística.

En relación con las líneas de investigación expuestas por este paradigma, la lingüística cognitiva persigue una estructura radial a modo de intersección integrativa con otras que ahonden en el fenómeno del lenguaje, más que llegar a concebirse como única posibilidad; en ese sentido, señala dos enfoques, a saber: las teorías generales, las cuales desarrollan conceptos generales y que son aplicables de manera concreta a los aspectos de las lenguas $\mathrm{y}$, los modelos gramaticales, que buscan elaborar un sistema panorámico y articulado del abordaje del lenguaje.

En la actualidad, la lingüística cognitiva es una corriente propia del vasto campo de las ciencias del lenguaje, con un objeto de estudio definido, unos principios teóricos y metodológicos pertinentes, unas tendencias establecidas, unas tareas delimitadas y unos alcances bien constituidos, donde el lenguaje es concebido como un fenómeno integrado dentro de las capacidades cognoscitivas del hombre.

\section{Fundamentos teóricos y metodológicos de la lingüística cognitiva}

Como se mencionó, la lingüística cognitiva propuso un cambio paradigmático en lo referente a las dos tradiciones filosóficas preponderantes: el objetivismo y el experiencialismo y su relación con la realidad y el lenguaje; entonces, desde una consideración real netamente objetiva, marcada por la veracidad o falsedad absoluta se optó por una formulación experiencial, donde "el lenguaje e incluso el mundo tienen significado solo y exclusivamente porque los seres humanos se lo confieren a través de la referida interacción" (Mairal, 2012, p. 139) y esto acaece debido a la capacidad integradora en la cognición humana.

Desde esta visión, y de acuerdo con Ibarretxe y Valenzuela (2012), uno de los axiomas más significativos de este nuevo paradigma lingüístico es el que tiene que ver con la concepción del lenguaje en tanto capacidad integradora y 
relacional de las tareas cognoscitivas humanas. Con lo expuesto se evidencia que el lenguaje no constituye una facultad cognitiva autónoma, sino que este va a depender de otras posibilidades relacionales entre las cuales sobresalen las cognitivas de percepción, conceptualización, categorización y las sensoexperienciales y desde luego su correlación en la aprehensión y representación tanto de la realidad como del mundo.

En ese orden de ideas, y según lo expresado por Ibarretxe y Valenzuela (2012), la lingüística cognitiva formula como principios teóricos fundamentales los siguientes: el lenguaje no es una facultad autónoma, independiente y distinta del resto de los procesos cognoscitivos y senso-perceptivos, lo que conlleva al rechazo del modularismo e innatismo (a). Todo concepto está basado en una estructura denominada dominio conceptual, es decir, todo concepto debe estar cimentado en una estructura dada por la experiencia (b). El lenguaje humano es dado a la categorización y prototipización en cuanto la información compartida (c). La relevancia del aspecto semántico del lenguaje en las tareas de análisis y descripción de fenómenos lingüísticos cobra una importancia esencial, puesto que este es fundamental en el abordaje del lenguaje (d). No es posible establecer una separación categórica entre el significado lingüístico y el significado extra lingüístico (e). La corporeización, comprendida como la configuración corporal capaz de la elaboración de categorías a partir del aparato sensomotor humano (f), y el lenguaje, basado en su uso regular en relación con la realidad del sujeto (g).

Adicional a esto, la lingüística cognitiva dentro de su elaboración investigativa, como se señaló anteriormente, propone unas teorías generales y unos modelos gramaticales para abordar el estudio del lenguaje. En relación con las teorías generales se encuentra lo propio a la teoría de prototipos, la semántica cognitiva y la teoría de la metáfora, lo que va a constituir la base epistemológica del cognitivismo lingüístico.

En cuanto a la teoría de prototipos, siguiendo a Cuenca (2013), se basa en un proceso de categorización no tradicional de conceptos dados por las relaciones lexicales que guardan a nivel de semejanza de familia y de constitución prototípica y periférica. En lo que tiene que ver con la semántica cognitiva, esta busca una interacción entre el significado denotativo y enciclopédico desde una aproximación pragmática, que dé razón de la relación entre lo convencional y lo no convencional del lenguaje. Con la teoría de la metáfora se pretende concebir a la metáfora más allá de un tropo literario y se le otorga una función cognitiva mediante la cual se llevan a cabo procesos mentales de procesamiento de información abstracta a partir de conceptos concretos.

Ahora bien, en cuanto al tratamiento de la gramática, en la lingüística cognitiva se ha orientado desde dos ópticas: la gramática cognitiva y la gramática de construcciones. La primera, siguiendo a Langacker (1991) citado por Cuenca (2013), señala que la gramática “... es un conjunto organizado de unidades simbólicas -resultado de la asociación de un polo semántico y un polo fonológico-". (p. 24), donde forma y función van juntas; es decir, no se concibe que 
la forma se dé independiente del significado ni de la función, lo que evita una disección separatista entre significado y sentido, al igual que la posibilidad de una modificación de la implicación misma del contenido que se comunica.

La gramática cognitiva por su parte pretende explicar las estructuras idiosincráticas de la lengua en cuanto relaciones simbólicas y relaciones convencionales entre significantes y significados, lo que otorga un valor simbólico a los recursos lingüísticos y a su vez permite una gama de posibilidades de comunicación, en tanto que un enunciado puede ser comunicado con diversas alternativas lingüístico-gramaticales, y en ese sentido, la gramática cognitiva busca la integración y construcción de unidades cognitivas y simbólico-funcionales del lenguaje desde diversas maneras de percibir la realidad.

Por lo tanto, desde los postulados expuestos, la lingüística cognitiva, como paradigma, se enmarca dentro de los campos epistémicos cognitivos en sí en cuanto a la relación integrativa que se teje entre lenguaje, cerebro y realidad.

\section{Principales tendencias de la lingüística cognitiva}

Dentro de las principales tendencias, en lo que atañe al tratamiento del lenguaje, concebido como fenómeno relacional integrado dentro de los procesos de cognición del hombre, la lingüística cognitiva propone las siguientes teorías: Teoría de la metáfora y la metonimia conceptual (a). La gramática cognitiva (b). La teoría de los espacios mentales (c) y la semántica de marcos (d).

En relación con la teoría de la metáfora y la metonimia conceptual (a), Ibarretxe y Valenzuela (2012) señalan que fue iniciada por Lakoff y Johnson, quienes "se dieron cuenta de que existían un gran número de expresiones lingüísticas que tomaban vocabulario de un dominio o área semántica aunque en realidad, su significado había que encontrarlo en otro dominio o área semántica distinta" (p. 24), lo que implicaba hacer uso del conocimiento previo de un campo conceptual para poder estructurar otro. Así se concibe una forma de pensamiento no literal, evidenciado también en el uso de la metonimia conceptual, en la cual un dominio conceptual es proyectado sobre otro, en lo respectivo a fuente y meta ${ }^{1}$. Con lo que se puede establecer que tanto la metáfora como la metonimia conceptuales son fenómenos mucho más que lingüísticos en sí y por ende se enmarcan en los procesos de estructuración y cognición en lo referente a dominios cognitivos, funciones referenciales y no referenciales y proyecciones conceptuales en términos relacionales dados entre el lenguaje, el pensamiento y la realidad.

En lo que tiene que ver con la gramática cognitiva (b), como se explicaba anteriormente, a diferencia de otras tradiciones lingüísticas, esta otorga un énfasis especial al significado dentro del análisis lingüístico, pues este componente es

1 "El primero se denomina dominio fuente, puesto que es el origen de la estructura conceptual que importamos. El segundo se denomina dominio meta o destino" (Soriano, 2012, p. 97). 
crucial en el tratamiento de la lengua, ya que todos los aspectos gramaticales traen consigo una carga significativa relevante; esto se debe a la corporeización del significado a través de la experiencia, el cual es procesado mediante la percepción y la cognición. Asimismo, en la gramática cognitiva se tienen en cuenta tres tipos de unidades: las semánticas, las fonológicas y las simbólicas. Maldonado (2012) señala que "un rasgo común de distintas escuelas cognitivas es reconocer en toda formación lingüística cargas específicas de significado asociadas a modos de significación" (p. 213). Así, en la lingüística cognitiva el significado es asimilado a la conceptualización en cuanto procesamiento cognitivo, puesto que:

Tanto en los morfemas cuanto en las construcciones sintácticas se reconoce una importante carga de sentido, de manera tal que la función lingüística no solo consiste en hacer evidente dicho significado, sino también en definir los patrones cognitivos que imperan en una y otra manifestaciones lingüísticas (Maldonado, 2012, p. 213).

Con lo cual queda por sentado el presupuesto que dentro del abordaje gramatical, el componente semántico muestra una gran importancia.

Ahora bien, en lo que se refiere a la teoría de los espacios mentales (c), señala Fauconnier (1994) que son "estructuras conceptuales parciales de realidades posibles que se activan de forma dinámica cuando se escucha un discurso o se lee un texto" (p. 148). Su característica específica radica en su función moldeadora, la cual no es más que la posibilidad de crear. La particularidad de estos está en que representan realidades posibles en lugar de la realidad concreta y por ende no pueden ser definidos como verdades objetivas; esto porque, siguiendo a Fauconnier (1994), los espacios mentales no son de naturaleza lingüística como tal, sino que estos reflejan operaciones cognoscitivas mucho más generales, en otras palabras, logran una amalgama ficción-realidad, a partir de las relaciones existentes entre lo uno y lo otro.

En lo concerniente a los marcos semánticos (d), Fillmore (citado por Ibarretxe y Valenzuela, 2012) sostiene que "para caracterizar de manera apropiada el significado de los elementos lingüísticos, como por ejemplo, las palabras, debemos primero conocer las estructuras conceptuales subyacentes en las que se basan y que les proporcionan un contexto mínimo e imprescindible para su comprensión" (p. 31); es decir, para conceptualizar una palabra se requiere de un marco que sirva de base para encontrar sus componentes constitutivos, lo cual es posible debido a los significados e implicaciones que a través de ellos se esquematizan en situaciones concretas. De esta forma, una misma palabra puede ostentar diversos significados a partir del contexto situacional donde se emplee, esto es porque para Fillmore, un marco es una categoría a través de la cual se organiza tanto la experiencia como la comprensión y explicación del significado, bien lexical, bien gramatical. 


\section{La lingüística cognitiva como paradigma lingüístico funcional y experiencial en lo referente al abordaje del lenguaje como fenómeno cognitivo integrado}

Con lo señalado hasta este punto se puede anotar la forma en la cual la lingüística cognitiva llega a constituirse en un paradigma lingüístico funcional y experiencial, al presentarse como una propuesta donde el lenguaje es pensado como un fenómeno cognitivo y perceptivo integrado y relacional. A continuación se presentan algunas proposiciones que permiten visualizar, a modo de reflexiones conclusivas, el porqué de dicho fenómeno.

En primer lugar y desde este paradigma lingüístico, el lenguaje, como fenómeno cognitivo integrado, se concibe desde una óptica en la cual los procesos de cognición y razonamiento dados a partir de la experiencia corporeizada estructuran las categorías de significado de la realidad a través de procesos de percepción, cognición, categorización, elaboración e integración conceptual y por ende de representación y referenciación del mundo. Esto en gran medida debido a "que nuestros conceptos, nuestras ideas, están influidos y conformados por la estructura de nuestros cuerpos, por nuestra experiencia del mundo que nos rodea" (Hilferty et al., 2012, p. 41).

En segundo lugar, las tareas cognoscitivas, en las cuales el lenguaje tiene asidero, son dadas a partir de procesos de pensamiento relacional integrativo, es decir, se originan mediante actividades complejas que involucran trabajos experienciales tanto a nivel mental como corpóreo de índole previo y de talante nuevo, los cuales facilitan la organización y almacenamiento de conocimientos sobre el mundo y la realidad y por ende de lo propio de la producción y comprensión lingüística.

Así, la lingüística cognitiva, como paradigma lingüístico funcional y experiencial en lo referente al abordaje del lenguaje como fenómeno cognitivo integrado, lo toma como instrumento de conceptualización, de cognición y de expresión de significados, el cual ostenta una doble naturaleza, la simbólica y la cognitiva; es decir, a través de éste no solo se aprehende la realidad sino que se le otorga sentido y esto debido a la capacidad relacional lenguaje-cognición, experiencia y mundo propia del ser humano.

En suma, la lingüística cognitiva al concebir el lenguaje como "aspecto del procesamiento cognitivo que puede definirse en términos de "patrones recurrentes de activación neuronal"'" (Cucatto, 2009, p. 16) y que simboliza, a través de las estructuras lingüísticas (Langacker, 1987, 1991), el modo en que los sujetos percibimos, experimentamos y categorizamos el mundo (a partir de experiencias individuales como así también colectivas o sociales), y, consecuentemente, le otorgamos significación (Pérez y Rojas, 2012, p. 2), pone de manifiesto que su 
paradigma permite entonces una relación integrativa funcional y experiencial en cuanto lenguaje, pensamiento y realidad.

\section{Referencias}

Cisneros, M. (1999). Reseña de Cuenca, M., y Hilferty, J. (1999). Introducción a la lingüística cognitiva. Barcelona, España: Ariel. Recuperado de http://cvc. cervantes.es/lengua/thesaurus/pdf/54/TH_54_001_356_0.pdf

Croft, W. (2008). Lingüística Cognitiva. Madrid, España: Ediciones Akal, S. A. Recuperado de http://www.kilibro.com/en/book/preview/36972/ linguistica-cognitiva

Cuenca, M., y Hilferty, J. (2013). Introducción a la Lingüística Cognitiva. Barcelona, España: Ariel.

Fauconnier, G. (1994). Mental spaces: Aspects of meaning construction in natural language. Cambridge University Press.

Hilferty, et al. (2012). Semántica cognitiva. Lingüística cognitiva. Barcelona, España: Anthropos.

Ibarretxe, I., y Valenzuela, J. (2012). Lingüística cognitiva. Barcelona, España: Anthropos Editorial.

Mairal. R., et al. (2012). Teoría lingüística. Métodos herramientas y paradigmas. Madrid, España: Editorial universitaria Ramón Areces UNED.

Maldonado, R. (2012). La gramática cognitiva. Lingüística cognitiva. Barcelona, España: Anthropos.

Pérez, L., y Rojas, G. (2012). Aportes de la lingüística cognitiva al análisis de discurso del periodismo audiovisual. I congreso de periodismo y medios de comunicación. Recuperado de http://www.textosenlinea.com.ar/monografias/ Aportes\%20de\%20la\%20linguistica\%20cognitiva\%20al\%20analisis\%20 del\%20discurso\%20del\%20periodismo\%20audiovisual.pdf

Soriano, C. (2012). La metáfora conceptual. Lingüística cognitiva. Barcelona, España: Anthropos. 